\title{
Perioperative Management of Case of Gynecological Malignancy with Bilateral Deep Venous Thrombosis of Lower Limbs Along with Pulmonary Embolism
}

\author{
Mahesh Kumar Arora ${ }^{1}$, Rakesh Garg ${ }^{2}$ \\ ${ }^{1}$ Department of Anaesthesiology and Intensive Care, All India Institute of Medical Sciences, New Delhi, India; ${ }^{2}$ Department of An- \\ aesthesiology and Intensive Care, All India Institute of Medical Sciences, New Delhi, India. \\ Email: drrgarg@hotmail.com
}

Received September $5^{\text {th }}$, 2011; revised October $10^{\text {th }}$, 2011; accepted October $18^{\text {th }}, 2011$.

\begin{abstract}
We describe the anaesthetic management of 45 year female patient with pre-existing deep venous thrombosis (DVT) and pulmonary embolism (PE) who was subsequently scheduled for a laparotomy. Before planning the surgical procedures, adequate anticoagulation must be achieved to prevent further complications of DVT, thromboembolism, and pulmonary embolism in particular. The risk of stopping the anticoagulation prior to surgery must be considered and adequately discussed with the patient and surgeons. The anaesthetic plan must be selected keeping in mind the coagulation status and the need of anticoagulation in the postoperative period.
\end{abstract}

Keywords: Deep Venous Thrombosis, Pulmonary Embolism, Anaesthesia, Oncology Surgery

\section{Introduction}

Venous thromboembolism (VTE), in the form of deep vein thrombosis (DVT) or pulmonary embolus (PE) is one of the concerns mostly in the postoperative period [1]. The presence of preexisting DVT and PE in patient scheduled for oncologic surgery carries a challenge for anaesthesiologist. As many as $50 \%$ of patients who have a proximal DVT develop a PE, and among those who have a clinically significant PE, $70 \%$ have evidence of DVT [1]. The underlying etiology for prothrombotic tendency of malignancies is the impact that malignant disease has on the Virchow's triad of stasis, intimal injury, and hypercoagulability [1]. Patients who have malignancy have a six fold increased risk of VTE, and it is estimated that the incidence of VTE in the cancer population is 1 in 200 patients [1-4]. Patients who have brain tumors and adenocarcinoma of the ovaries, pancreas, stomach, colon, lung, prostate, and kidney have a higher risk of VTE than those who have other malignancies [4-6]. With other risk factors equal, surgery for malignant disease results in a 2- to 3-fold increase in thromboembolism compared with surgery for nonmalignant conditions [1,5]. Among the all malignancies, the gynecologic oncology patients have the highest risk of venous thromboembolism [6]. Though postoperative DVT and PE usually remains a concern and various measures are mentioned in literature for its prevention but the presence of DVT and PE preoperatively in a patient scheduled for oncology surgery and its perioperative management has not been much described in literature.

We present a case of bilateral lower limb DVT with pulmonary embolus scheduled for laparotomy for gynaecology malignancy.

\section{Case History}

A 45-year-old female weighing $72 \mathrm{~kg}$ was scheduled for laparotomy for the excision of abdominoplevic mass and carcinoma ovary. On reviewing the history, patient had fever for last 3 weeks, pain and swelling in both lower limbs since last 10 days. The swelling appeared in the foot and gradually involved the whole of the both lower limbs in 3 - 4 days. Doppler examination of lower limb revealed bilateral deep venous thrombosis (DVT). She was started on inj enoxaparin $0.6 \mathrm{~mL}$ once daily since 4 days. Two days back she complained of breathlessness. 
She was referred to our institute. On examination, her pulse rate was 92 beats per min, blood pressure of 108/82 $\mathrm{mmHg}$ and respiratory rate was $22 / \mathrm{min}$. Investigations revealed haemoglobin $8.4 \mathrm{~g} / \mathrm{dL}$. Her D-Dimer by quantitative Immunoturbidimetry method was $>20 \mu \mathrm{gFibEq} / \mathrm{mL}$ (normal < 0.5). Computed tomographic angiogram revealed thrombus in left descending pulmonary artery with extension into left lower lobe, infrarenal inferior vena cavae (IVC), bilateral common iliac vein, external iliac vein, common femoral vein, saphenofemoral vein, popliteal veins along with hepatomegaly and abdominopelvic mass encasing IVC. Haematologists opinion was taken and dose of inj enoxaparin was increased to $80 \mathrm{mg}$ sc twice a day. The risk associated with the surgery and written informed consent was taken. IVC filter was placed in the infrarenal IVC via right transjugular approach.

The dose of enoxaparin was omitted on the morning of surgery and patient was premedicated with oral diazepam (10 mg) and pantaprazole (40 mg). Her platelet counts were $180 \times 10^{3} / \mathrm{mm}^{3}$, prothrombin time-13:17 (control:test), aPTT - 28:30 (control:test). In the operating room, routine monitors were attached. Anaesthesia was induced with intravenous fentanyl $(150 \mu \mathrm{g})$, propofol $(140 \mathrm{mg})$ and lungs ventilated with oxygen in isoflurane (2\%). After achieving neuromuscular blockade with atracurium (40 $\mathrm{mg}$ ), trachea was intubated with endotracheal tube. Left radial artery was cannulated. Ultrasound guided right IJV was cannulated using triple lumen central venous catheter (CVC). Anaesthesia was maintained with isoflurane in oxygen and air along with infusions of fentanyl $(50 \mu \mathrm{g} / \mathrm{hr})$ and atracurium (20 mg/hr). Intraoperatively tumor mass was excised and blood loss was $1500 \mathrm{~mL}$, which was replaced with $1500 \mathrm{~mL}$ of voluven initially and then with 4 units of packed RBC (after removal of tumor mass). Patient remained haemodynamically stable. After completion of surgery, residual neuromuscular blockade was reversed and trachea was extubated. She was shifter to ICU for observation. Inj enoxaparin was restarted 6 hours later. Analgesia was provided with intravenous morphine infusion $(1 \mathrm{mg} / \mathrm{hr})$. She remained stable and shifted to ward on $2^{\text {nd }}$ day and discharged home on the $8^{\text {th }}$ day uneventfully.

\section{Discussion}

A number of issues related to pre-existing DVT prior to gynecologic surgery deserve further clarification, the optimal duration of pharmacological treatment, and the optimal duration/modality of prophylaxis to prevent thromboembolism in these high-risk cancer patients. The other concern was bleeding during the perioperative period due to anticoagulation for treatment of DVT and pro-coagulation associated with the malignancy. The pathogenesis of perioperative PE is assumed to be based on a hypercoagulable state that may occur during major surgery and that may extend into the postoperative period [7]. Massive PE is associated with haemodynamic instability and high mortality (30\%). So, the treatment options include anticoagulation, thrombolysis, placement of a vena caval filter and pulmonary embolectomy [8]. Further the thrombus extension occurs during the immobility in the perioperative period. This chance of thrombus extension and risk of further pulmonary embolus in our patient having bilateral DVT prompted us to insert IVC filter prophylactically. Placement of IVC filter prior to urgent surgery in a patient with a DVT has also been described earlier [5,9]. The use of vena caval filters has expanded to include primary venous thromboembolism (VTE) prophylaxis in special patient populations such as major trauma patients, major surgery patients, advanced malignancy, and neurological or neurosurgical patients with paralysis or prolonged immobilization. We were apprehensive about the dislodgement of IVC filter intraoperatively as site of its placement was as same as surgical site. Surgeons were careful enough to avoid compression or distortion of the IVC.

We started the low molecular weight heparin (LMWH) - enoxaparin in our patient as LMWH like enoxaparin, dalteparin, tinzaparin are considered the initial treatment of choice, and may be preferable for patients with cancer $[1,5,10,11]$. LMWHs are as effective as unfractionated heparin (UFH) for reducing DVT recurrence, thrombus extension and risk of death due to PE. For acute VTE treatment, limitations of UFH include an unpredictable anticoagulant response with the need for frequent monitoring, a relatively narrow therapeutic window, and the potential for severe toxicity, especially heparininduced thrombocytopenia (HIT) [12]. Importantly, trials of UFH in PE show that many patients are subtherapeutic or supratherapeutic while receiving UFH. In contrast, LMWHs provide a more targeted approach to procoagulant complex inhibition, predictable pharmacokinetic and pharmacodynamic characteristics, and no need for anticoagulant monitoring [12]. LMWH is the preferred approach for the initial 5 to 10 days of anticoagulant treatment of the cancer patient with established VTE $[2,13]$. LMWHs are typically given subcutaneous in a standard weight-based dose (eg, enoxaparin $1.5 \mathrm{mg} / \mathrm{kg}$ sc once/day or $1 \mathrm{mg} / \mathrm{kg}$ sc twice a day or dalteparin 200 units $/ \mathrm{kg}$ sc once a day). LMWH is considered to be effective for DVT management in patients with cancer. Thrombolytic therapy of DVT is generally reserved for patients who have limb-threatening thrombosis, symptoms for less than a week and low risk of bleeding. Absolute indications for thrombolytic therapy are a massive 
iliofemoral DVT, especially in the case of phlegmasia cerulea dolens, and a pulmonary embolism that is accompanied by hemodynamic instability. Our patient had a history of DVT for 3 weeks and was stable hemodynamically, so we avoided thrombolysis when the patient presented to us and started her on enpxaparin. Nevertheless, thrombolytics have not been shown to decrease mortality in these patients.

The quantitative rapid ELISA, with a sensitivity of $95 \%$, in general has shown the most clinically useful values among the various D-dimer assays [13]. An abnormal D-dimer indicates the need for further testing if $\mathrm{PE}$ is suspected. The imaging modalities includes contrast enhanced multidetector computerized tomography pulmonary angiography (CT angiography) and CT venous phase imaging of the proximal leg veins (CT venography). The gold standard for the diagnosis of $\mathrm{PE}$ is Contrast pulmonary arteriography. In the presence of breathlessness, we suspected PE and since D-Dimers were raised, we confirmed the presence of PE with CT angiography.

The patients with PE, the gas-exchange abnormalities related to various variables including the size and character of the embolic material, the extent of the occlusion, the underlying cardiopulmonary status and the length of time since embolization [13]. Hypoxemia has been attributed to an increase in alveolar dead space, right-to-left shunting, ventilation/perfusion inequality, and a low mixed venous oxygen level. Classic triad of PE dyspnea, chest pain and hemoptysis is seen in $20 \%$ of patients. Our patient had only breathlessness and preoperative ABG was within normal limits. This may probably be because of thrombosis only in one segment of lung.

So, we conclude, great vigilance is required in the management of a patient with bilateral lower limb deep venous thrombosis with pulmonary embolism. Measures for prevention of further pulmonary embolism should be undertaken prior to urgent oncology surgery. Before planning the surgical procedures, adequate anticoagulation must be achieved to prevent further complication of DVT, thromboembolism, and pulmonary embolism. The risk of stopping the anticoagulation prior to surgery must be considered and adequately discussed with the patient and surgeons. The anaesthetic plan must be selected keeping in mind the coagulation status and the need of anticoagulation in the postoperative period.

\section{REFERENCES}

[1] D. A. Anaya and A. B. Nathens, "Thrombosis and Coagulation: Deep Vein Thrombosis and Pulmonary Embolism Prophylaxis,” The Surgical Clinics of North America, Vol. 85, No. 6, 2005, pp. 1163-1177. doi:10.1016/j.suc.2005.10.015
[2] G. H. Lyman, A. A. Khorana, A. Falanga, D. C. Pearson, C. Flowers, M. Jahanzeb, A. Kakkar. et al., "American Society of Clinical Oncology Guideline: Recommendations for Venous Thromboembolism Prophylaxis and Treatment in Patients With Cancer," Journal of Clinical Oncology, Vol. 25, No. 34, 2007, pp. 5490-5505. doi:10.1200/JCO.2007.14.1283

[3] N. Levitan, A. Dowlati, S. C. Remick, H. I. Tahsildar, L. D. Sivinski, R. Beyth, et al., "Rates of Initial and Recurrent Thromboembolic Disease among Patients with Malignancy versus Those without Malignancy,” Medicine, Vol. 78, No. 5, 1999, pp. 285-291. doi:10.1097/00005792-199909000-00001

[4] J. W. Blom, C. J. M. Doggen and S. Osanto, "Malignancies, Prothrombotic Mutations, and the Risk of Venous Thrombosis," The Journal of the American Medical Association, Vol. 293, No. 6, 2005, pp. 715-722. doi:10.1001/jama.293.6.715

[5] S. M. Bates and J. S. Ginsberg, "Treatment of Deep-Vein Thrombosis," New England Journal of Medicine, Vol. 351, 2004, pp. 268-277. doi:10.1056/NEJMcp031676

[6] M. A. Martino, E. Borges, E. Willianson, S. Siegfried, A. B. Cantor, J. Lancaster, et al., "Pulmonary Embolsim after Major Abdominal Surgery in Gynecologic Oncology,” Obstetrics \& Gynecology, Vol. 107, No. 3, 2006, pp. 666-671. doi:10.1097/01.AOG.0000200046.28199.ae

[7] T. B. Comfere, J. Sprung, K. A. Case, P. T. Dye, J. L. Johnson, B. A. Hall, et al., "Predictors of Mortality Following Symptomatic Pulmonary Embolism in Patients Undergoing Noncardiac Surgery,” Canadian Journal of Anesthwsia, Vol. 54, No. 8, 2007, pp. 634-641. doi:10.1007/BF03022957

[8] P. Rosenberger, S. K. Shernan, P. S. Shekar, J. K. Tuli, T. Weissmuller, S. F. Aranki, et al., "Acute Hemodynamic Collapse after Induction of General Anesthesia for Emergent Pulmonary Embolectomy,” Anesthesia \& Analgesia, Vol. 102, No. 5, 2006, pp. 1311-1315. doi:10.1213/01.ane.0000208970.14762.7f

[9] K. M. Sutin, C. Schneider, N. S. Sandhu and L. M. Capan, "Deep Venous Thrombosis Revealed during UltrasoundGuided Femoral Nerve Block,” British Journal of Anaesthesia, Vol. 94, No. 2, 2005, pp. 247-248. doi:10.1093/bja/aei036

[10] V. Snow, A. Qaseem, P. Barry, E. R. Hornbake, J. E. Rodnick, T. Tobolic, et al., "Management of Venous Thromboembolism: A Clinical Practice Guideline from the American College of Physicians and the American Academy of Family Physicians," Annals of International Medicine, Vol. 146, No. 3, 2007, pp. 204-210.

[11] J. B. Segal, M. B. Streiff, L. V. Hofmann, K. Thornton and E. B. Bass, "Management of Venous Thromboembolism: A Systematic Review for a Practice Guideline," Annals of International Medicine, Vol. 146, No. 3, 2007, pp. 211-222.

[12] A. C. Spyropoulos, "Investigational Treatments of Venous Thromboembolism,” Expert Opinion Investigational Drugs, Vol. 16, No. 4, 2007, pp. 431-440. doi:10.1517/13543784.16.4.431 
[13] P. D. Stein, R. D. Hull, K. C. Patel, et al., "D-dimer for the Exclusion of Deep-Venous Thrombosis and Acute Pulmonary Embolism: A Systematic Review,” Annals of
International Medicine, Vol. 140, No. 8, 2004, pp. 589602. 